\title{
Dietary glycaemic index and glycaemic load in Danish children in relation to body fatness
}

\author{
Birgit M. Nielsen ${ }^{1,2,3 *}$, Kirsten S. Bjørnsbo ${ }^{1,2}$, Inge Tetens ${ }^{3}$ and Berit L. Heitmann ${ }^{1}$ \\ ${ }^{1}$ Research Unit for Dietary Studies, Institute of Preventive Medicine, Copenhagen University Hospital, Denmark \\ ${ }^{2}$ European Youth Heart Study, Institute of Sports Science and Clinical Biomechanics, University of Southern Denmark, Odense, Denmark \\ ${ }^{3}$ Department of Human Nutrition, Centre for advanced Food Studies, The Royal Veterinary and Agricultural University, Frederiksberg, \\ Denmark
}

(Received 23 August 2004 - Revised 10 February 2005 - Accepted 10 February 2005)

\begin{abstract}
The aim of this study was to describe dietary glycaemic index (GI) and glycaemic load (GL) values in the diets of Danish children, and to examine the associations between dietary GI, GL and body fatness. Data were collected during 1997-8 as part of the European Youth Heart Study. The study population comprised 485 children aged 10 years and 364 children aged 16 years from Odense County, Denmark. Dietary GI and GL were estimated using international food tables, and the associations between energy-adjusted dietary GI, GL and body fatness were analysed by multiple linear regression. The mean daily dietary GI value was 85 (SD 6.9) with a range of 62-111. No significant differences were found between age groups and gender. The daily dietary GL was higher among boys aged 16, with a GL of 330 (SD 95) $(P<0.05$ ), compared with girls or younger boys. Dietary GL was higher among 10-year-old boys than girls ( 250 (SD 81$)$ v. 230 (SD 66) $P<0.05$ ), whereas dietary GL among 16-year-old girls was 230 (SD 56). Neither dietary GI nor GL was associated with the sum of four skinfolds ( $\Sigma \mathrm{SF}$ ) among girls or among 10-year-old boys. Among 16-year-old boys, significant associations were observed between dietary GI and $\Sigma \mathrm{SF}(\beta=0 \cdot 60, \mathrm{SE}=0 \cdot 21, P=0 \cdot 006)$, and between dietary GL and $\Sigma \mathrm{SF}(\beta=0 \cdot 15, \mathrm{SE}=0.06, P=0 \cdot 009)$. In conclusion, dietary GI and GL were positively associated with body fatness among Danish boys aged 16 years, whereas no associations were found among girls or younger boys.
\end{abstract}

Children: Adolescents: Diet: Body fat mass

The prevalence of obesity is increasing rapidly worldwide. Obesity in childhood is of special concern because of the wide range of severe complications already present in childhood and the increased risk of premature illness and death in adulthood. Furthermore, the excessive weight in childhood is likely to persist into adulthood (World Health Organization, 2003). The search for possible determinants of the development of obesity in childhood is thus of great interest and importance; in addition, diet, physical activity, genetic factors and social influences are some of the main studied contributors to the development of excessive body weight (World Health Organization, 2003). Many dietary factors have been investigated, particularly the influence of the percentage of energy from dietary fat (Ludwig, 2000; Pawlak et al. 2002), but evidence for an association among children as well as adults is lacking (Lissner \& Heitmann, 1995).

One newly proposed dietary determinant of body composition, including obesity, is glycaemic index (GI), which is a ranking of carbohydrate-containing foods according to their postprandial blood glucose response (Augustin et al. 2002; Ludwig, 2002). A low-GI diet is hypothesised to increase satiety and reduce voluntary food intake, whereas a high-GI diet is expected to increase hunger and voluntary food intake, and in turn increase the accumulation of fat on the body (Augustin et al. 2002; Ludwig, 2002; Pawlak et al. 2002). In a cross-over design among overweight men with 5-week periods of low-GI and high-GI diets, Bouché et al. (2002) found that body fat mass decreased and lean body mass increased while eating the lowGI diet, whereas the body composition remained stable through the period of eating the high-GI diet. Similarly, in an animal model, Pawlak et al. (2004) found a reduced fat mass in those animals eating a low-GI diet compared with those eating a high-GI diet.

With regard to children, only one study so far has reported on the association between BMI and dietary GI, and this study did not find any significant association (Scaglioni et al. 2004). Hence, the aim of the present study was to describe the GI and glycaemic load (GL) of the habitual diet of 849 Danish children, followed by an examination of the possible associations between dietary GI (or GL) and body composition.

\section{Materials and methods}

\section{Subjects}

The study population comprised 10- and 16-year-old children from Odense enrolled as part of the European Youth Heart Study. Odense is the third largest city in Denmark, with 180000 inhabitants. The study design, sampling and methods 
for the European Youth Heart Study have been described in more detail elsewhere (Riddoch et al. 2004a,b; Wedderkopp, 2001). In total, 1356 children were invited to participate in the study, of whom 1325 responded and 1020 agreed to participate (Wedderkopp, 2001). The collection of data was conducted during one whole school year, from 10 August 1997 to 12 July 1998 (Wedderkopp, 2001). In total, 849 of the participating children presented complete information on diet and anthropometrics, and were included in this study.

The Danish part of the European Youth Heart Study adhered to the Helsinki Declaration and was approved by the local ethics committee (Wedderkopp, 2001).

\section{Assessment of dietary intake}

Information on dietary intake was obtained by a $24 \mathrm{~h}$ recall, supported by a qualitative food record. The children completed a qualitative food record at home, followed the next day by a face-to-face interactive interview carried out by the same interviewer for all children. Interviews were carried out on school days, so dietary intake was not obtained for Fridays and Saturdays. The quantity of foods eaten and meals was estimated using common household measures and food pictures (Riddoch et al. 2004a,b). Afterwards, the interviewer computed the dietary information in a database, by which it was possible to calculate nutrient information on the individual food items or whole meals and diets via the national food composition tables (Moller, 1996). The use of the $24 \mathrm{~h}$ recall method has been validated in children by Lytle et al. (1993), who concluded that the method was useful.

\section{Calculation of dietary glycaemic index and load}

The GI value and grams of available carbohydrate per gram of each food item were primarily assessed from the international table (Foster-Powell et al. 2002), GI being expressed as a percentage of the glycaemic response elicited by 'white bread'. This table does not provide GI values for meat, poultry, fish, cheese, eggs and most vegetables (exceptions being potato, sweetcorn, pumpkin, beetroot, carrots, parsnips and swede) owing to their low carbohydrate content and their subsequent negligible influence on the postprandial glycaemic response (Foster-Powell et al. 2002). Thus, a GI value of zero was assumed for these food items. Otherwise, when a GI value for a food item had not been determined in the literature, the GI value of the nearest comparable food item was assigned instead (e.g. a Danish pastry being assigned the GI value of a doughnut), or it was calculated from the constituent foods.

The GI value for the overall diet was calculated as described by Wolever \& Jenkins $(1985,1986)$. Briefly, for each subject, the daily overall dietary GI was calculated as the weighted average of the GI values of each of the foods consumed, the weights being based on the proportion of available carbohydrate contributed by each food item. Thus:

Individual overall dietary GI $(/ \mathrm{d})=\Sigma\{(\mathrm{GI}$ of food item $) \times$ (available carbohydrate content of food item (g)) / (total daily intake of available carbohydrate $(g))\}$

For each subject, the daily dietary GL was calculated by multiplying the overall dietary GI by the total daily intake of available carbohydrate content (g) (Salmeron et al. 1997a,b; Foster-Powell et al. 2002). Thus:
Individual daily dietary GL = (overall dietary GI)

$\times$ (total daily intake of available carbohydrate $(\mathrm{g})$ )

Each unit of daily dietary GL represents the equivalent of $1 \mathrm{~g}$ available carbohydrate from white bread (Brand-Miller et al. 2003).

\section{Measurement of non-dietary factors}

All measurements were carried out as standardised methods, which have been described in detail elsewhere (Riddoch et al. 2004a,b; Wedderkopp, 2001).

Body height was measured for each child to the nearest millimetre using a stadiometer, and body weight was measured to the nearest $100 \mathrm{~g}$ using a beam-scale-type weight (Council of Europe, 1988; Wedderkopp, 2001).

Skinfold thickness was obtained for each child twice on the left-hand side at four locations measured with a Harpenden calliper (John Bull; British Indicators Ltd, Birmingham, UK) over the triceps brachii and biceps brachii muscles, subscapularly, and superior to the anterior superior iliac spine (Slaughter et al. 1988; Wedderkopp, 2001), and finally expressed as the sum of the four skinfold thicknesses ( $\Sigma \mathrm{SF})$. The same male investigator made all the skinfold measurements on the boys, whereas two female investigators made all the measurements on the girls. When continuously checking the reliability of the skinfold measurements, inter- and intra-tester Pearson correlation coefficients of 0.988 and 0.998 were found between skinfold measurements (Wedderkopp, 2001).

Pubertal status was assessed by the investigators from a scale of pictures according to Tanner (1962) (Wedderkopp, 2001).

Physical fitness was determined by a maximal work test (wattmax test), performed as a progressive maximal cycle ergometer test (cycle ergometer Monark Ergomedic 839; Monark Exercise AB, Vanobro, Sweden), and physical fitness was calculated as the maximal power output (wattmax) (Wedderkopp, 2001). The wattmax test as well as the calculation of wattmax are described in detail by Brage et al. (2004). Both in previous validation studies among other groups of children (Hansen et al. 1989; Andersen, 1995) and in a validation performed for this study population, there was a good agreement between the wattmax test and directly measured $\mathrm{V}_{\mathrm{O} \text { 2max }}$ (Wedderkopp, 2001). The following equations, for calculating the fitness (relative $\mathrm{V}_{\mathrm{O} 2 \max }$ ) of each age group, were developed in this validation study (N Wedderkopp, personal communication) and used in the present study:

$$
\begin{aligned}
& \text { 10-year-olds : Fitness }\left(\mathrm{ml} \mathrm{O}_{2} /\right. \text { min per kg) } \\
& \qquad=(250 \cdot 26+12 \cdot 44 \text { wattmax }(\mathrm{W})) / \text { body weight }(\mathrm{kg})
\end{aligned}
$$

16-year-olds : Fitness $\left(\mathrm{ml} \mathrm{O}_{2} / \mathrm{min}\right.$ per $\left.\mathrm{kg}\right)$

$$
=(364.79+11.87 \text { wattmax }(\mathrm{W})) / \text { body weight }(\mathrm{kg})
$$

\section{Statistical analysis}

ANOVA and $t$ tests were performed to compare group means, using Tukey's procedure to adjust for multiple comparisons. Analysing homogeneity of variances was conducted using Levene's test. Groups of data that were non-normal or had unequal variances were analysed using Kruskal-Wallace and 

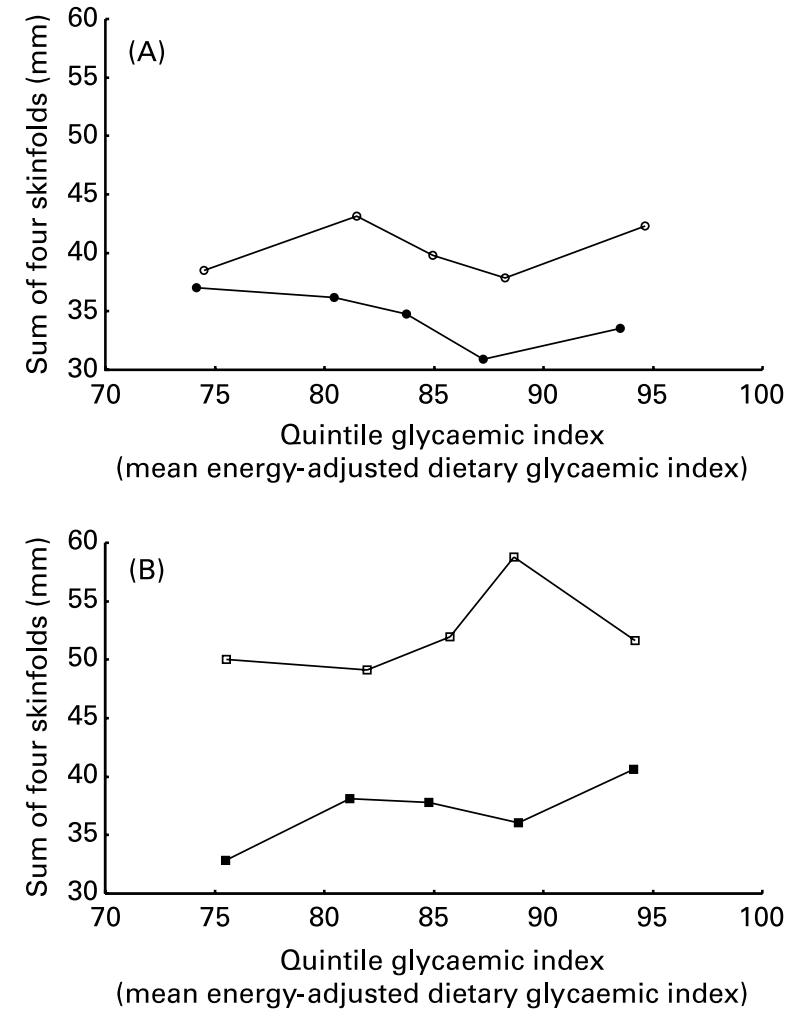

Fig. 1. Association between body composition and quintiles of energyadjusted dietary glycaemic index of children: (A) 10-year-old boys ( $\bullet$ ) and girls (O), and (B) 16-year-old boys ( $\square$ ) and girls ( $\square$ ).

Mann-Whitney tests, with Bonferroni's adjustment to control for multiple comparisons. Each dietary and anthropometric variable was treated as a continuous variable. Having started puberty was categorised as yes or no. To improve the normality of their distribution, $\Sigma \mathrm{SF}$ and weight were logarithmically transformed. To control for total energy intake in the backward multiple linear regression analyses, dietary GI, dietary GL and macronutrient intake were adjusted for total energy intake using the residual method (Willett \& Stampfer, 1986). All variables were logarithmically transformed before adjustments to assure non-correlation between residuals and total energy intake. Adjustments were made for energy-adjusted protein and fat intakes in all analyses of associations between dietary GI and body composition. In addition, other potential confounding factors, including weight, height, fitness and the start of puberty, were adjusted for in analyses of the associations between dietary GI (or GL) and body composition. Bonferroni corrections were applied to the backward multiple linear regressions. Model control showed no pattern in the residuals, and they had an approximately normal distribution.

A $P$-value $<0.05$ was considered as indicating statistical significance. All statistical analyses were performed using the SAS System, Version 8.2 (SAS Institute Inc., Cary, NC, USA).

\section{Results}

The characteristics of the children, grouped by age and gender, are shown in Table 1.

Across age and gender, children from the present study had a mean carbohydrate intake of $320 \mathrm{~g} / \mathrm{d}$ (value not energy adjusted), a GI value of which was assigned to $96 \%$ of this carbohydrate.

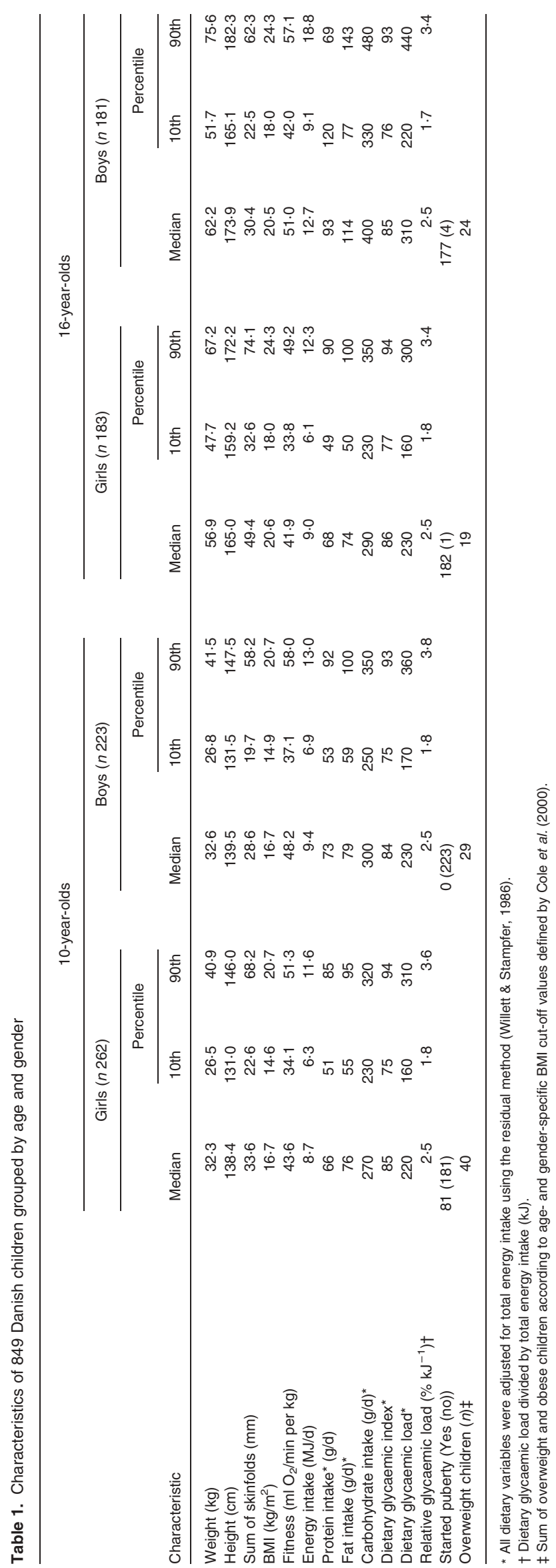


Thus, overall dietary GI and dietary GL in the present study reflected $96 \%$ of the total carbohydrate intake.

\section{Prevalence of overweight and obesity}

The prevalence of overweight and obesity was assessed according to the age- and gender-specific BMI cut-off values published by Cole et al. (2000). The total prevalence of overweight and obesity was $15 \%$ among girls and $13 \%$ among boys aged 10 years, whereas the prevalence among 16-year-olds was $10 \%$ among girls and $13 \%$ among boys.

\section{Distribution of overall dietary glycaemic index and glycaemic load}

Daily dietary GI was normally distributed within each group of age and gender, and no significant differences were found between groups $(P>0 \cdot 19)$. The value of energy-adjusted dietary GI ranged from 62 to 111, with a mean of 85 (SD 6.9).

The distribution of the daily dietary GL was skewed to the left, and was therefore $\log$-transformed. The log-transformed daily dietary GL was significantly higher among 16-year-old boys than others (all $P<0.0001$ ), with a mean energy-adjusted dietary GL value of 330 (SD 95). Energy adjusted dietary GL among 10year-old boys was significantly higher than among 10-year-old girls (250 (SD 80) v. 230 (SD 70); $P<0 \cdot 05$ ). The energy-adjusted dietary GL value among 16-year-old girls was 230 (SD 56).

Association between overall dietary glycaemic index, glycaemic load and body composition or BMI

Both before and after adjustment for the start of puberty, fitness and energy-adjusted protein and fat intake, there were no significant associations between energy-adjusted dietary GI (or GL) and $\Sigma$ SF among the girls in each age group, or among 10-year-old boys (all $P>0.05$ ). In the adjusted but not in the crude analyses, significant associations were observed between dietary GI (energy adjusted) and $\Sigma$ SF $(\beta=0 \cdot 60, \mathrm{SE}=0 \cdot 21, P=0.006)$, and between dietary GL (energy adjusted) and $\Sigma \mathrm{SF}(\beta=0 \cdot 15$, SE $=0.06$, $P=0.009$ ) among 16-year-old boys (Table 2 and Fig. 1). Thus, a difference in dietary GI of $10 \%$ was associated with a $6(\mathrm{SE} 2) \%$ higher $\Sigma \mathrm{SF}$, whereas a difference in dietary GL of $10 \%$ was associated with a 1 (SE 0.6$) \%$ higher $\Sigma$ SF among 16-year-old boys. Associations between (energy-adjusted) dietary GI (or GL) and BMI were insignificant among each group of age and gender (all $P>0.05$; results not shown). Substituting the adjustment for fat intake (energy adjusted) with an adjustment for carbohydrate intake (energy adjusted) in the above-mentioned analyses did not result in different results. Further adjustment for fibre intake (energy adjusted) did not alter the above results either and hence was not accounted for in the final model.

\section{Discussion}

In the present study, dietary GI was similar for Danish girls and boys aged 10 and 16 years, whereas 16-year-old boys had a higher daily dietary GL than both girls of a similar age and younger girls and boys. GI and GL were positively associated with body fatness among Danish 16-year-old boys, whereas no associations were found among girls or younger boys.

Except for Scaglioni et al. (2004), no other studies have previously published associations between dietary GI (or GL) and body fatness in children. Scaglioni et al. (2004) found no associations between dietary GI (or GL) and BMI among 8-year-olds. In agreement, our data did not show any significant associations between dietary GI (or GL) and BMI. As BMI reflects not only body fatness, but also the relative length of the legs, body frame size and fat-free body mass (Prentice \& Jebb, 2001), subjects with similar BMI do not necessarily have the same amount of body fat. However, skinfold thickness has previously been found to be a good indicator of body fatness in children (Sardinha et al. 1999; Wedderkopp, 2001) and a better predictor of percentage body fat than BMI (Sarría et al. 1998). Thus, the significant association between dietary GI (and GL) and body composition expressed as $\Sigma$ SF, but not as BMI, may be explained by the association being mediated by the amount of body fat rather than the degree of overweight. This would be in accordance with results from other studies, in which the consumption of low-GI diets compared with high-GI diets was associated with a smaller body fat mass and a larger lean body mass, but not with total body weight (Bouché et al. 2002; Pawlak et al. 2004). Both skinfold thickness and BMI are proxy measures of body composition, and a more valid measure of body fat mass (e.g. dual energy X-ray absorptiometry) may be needed to investigate further the possible association between dietary GI (and GL) and body fat mass.

Table 2. Association between dietary glycaemic index or glycaemic load and body composition expressed as the sum of four skinfold thicknesses of children grouped by age and gender

\begin{tabular}{|c|c|c|c|c|c|c|c|c|c|}
\hline & & \multicolumn{4}{|c|}{ 10-year-olds } & \multicolumn{4}{|c|}{ 16-year-olds } \\
\hline & & \multicolumn{2}{|c|}{ Girls $n 262$} & \multicolumn{2}{|c|}{ Boys $n 223$} & \multicolumn{2}{|c|}{ Girls $n 183$} & \multicolumn{2}{|c|}{ Boys $n 181$} \\
\hline & & $\beta$ & SE & $\beta$ & SE & $\beta$ & SE & $\beta$ & SE \\
\hline \multirow[t]{2}{*}{ Dietary glycaemic index $\ddagger$} & Crude model & 0.23 & 0.30 & -0.19 & 0.34 & 0.38 & 0.31 & 0.66 & 0.37 \\
\hline & Full model $A^{*}$ & 0.29 & 0.17 & -0.13 & 0.17 & -0.09 & 0.17 & $0.60 \S$ & 0.21 \\
\hline \multirow[t]{2}{*}{ Dietary glycaemic load } & Crude model & 0.29 & 0.09 & -0.10 & 0.09 & 0.05 & 0.10 & 0.15 & 0.11 \\
\hline & Full model B† & 0.08 & 0.05 & -0.02 & 0.05 & 0.05 & 0.05 & 0.159 & 0.06 \\
\hline
\end{tabular}

${ }^{*}$ Full model A. Adjustment: fitness $\left(\mathrm{ml} \mathrm{O} / \mathrm{min}\right.$ per $\mathrm{kg}$ ), $\log _{\mathrm{e}}$ weight, height $(\mathrm{m})$, protein intake $(\mathrm{g} / \mathrm{d}) \ddagger$, fat intake $(\mathrm{g} / \mathrm{d}) \ddagger$ and, among 10 -year-old girls, whether puberty had started (yes/no).

†Full model B. Adjustment: fitness ( $\mathrm{ml} \mathrm{O}_{2} / \mathrm{min}$ per $\mathrm{kg}$ ), $\log _{\mathrm{e}}$ weight, height $(\mathrm{m})$ and, among 10-year-old girls, whether puberty had started (yes/no)

†Adjusted for total energy by the residual method (Willett \& Stampfer, 1986).

$\S P=0.006$.

$P=0.009$ 
The positive association between GI (or GL) and body fat found among 16-year-old boys but not girls may depend on a larger diet-reporting bias on the part of the girls than the boys. Indeed, dietary reporting bias may vary between groups. For example, Bandini et al. (2003) found a considerable degree of under-reporting among girls as they matured from 10 to 15 years. In the present study, the mean energy intake of 10- and 16-year-old girls was almost the same despite the 6-year age difference and the greater weight and heights among the 16year-olds. This suggests an under-reporting by the older girls of their 'habitual' food intake. Under-reporting of the quantity of the diet will result in an underestimation of dietary GL, whereas dietary GI may not be systematically affected. On the other hand, if individual food items were either under- or over-reported, both dietary GI and GL might be affected. In the present study, we cannot predict whether this would systematically deflate or inflate the estimates of GI and GL; however, random misclassification is likely and would influence the results by attenuating the association with body fatness. Indeed, random misclassification may explain why we were unable to find significant associations for all groups of boys and girls.

On the other hand, different food consumption patterns may result in a similar GI. In the present study, the 16-year-old boys consumed relatively more milk and meat products, and less fruit, compared with the girls of similar age, whereas the intake of food groups in the diets of 10-year-old children was similar between boys and girls (data not shown). The implication of these different food consumption patterns among 16-year-old boys and girls may be that associations between food items or food groups and the dietary GI are different, and hence may explain why dietary GI and GL were associated with body fatness among the 16-year-old boys but not the girls.

No previous Danish study has published data on the GI or GL of the habitual diet of children, adolescents or adults. However, as in the present study, Scaglioni et al. (2004) found that mean dietary GI was similar across gender (Danish GI 85 (SD 7) $v$. Italian GI 83 (SD 3)) and that boys had a significantly higher dietary GL than girls, in agreement with a higher carbohydrate intake among boys (Scaglioni et al. 2004). In contrast, the mean dietary GL value (energy adjusted) of Danish children was nearly $24 \%$ higher than the Italian mean (Danish GL 256 (SD 85) v. Italian GL 207 (SD 53)). Scaglioni et al. (2004) assessed dietary intake by a food-frequency questionnaire, whereas this study used a $24 \mathrm{~h}$ recall. The dietary assessment method used will influence the assessment of GL and may very well explain the observed difference between the Italian and Danish data for GL, suggesting that comparisons of GL between different studies should be carried out with caution.

The few studies of dietary GI conducted within normal adult population groups have yielded ranges similar to those of the present study on children, whereas dietary GL among healthy adults seems somewhat lower than the values found in this study (Salmeron et al. 1997a,b; Liu et al. 2000, 2002; van Dam et al. 2000; Levi et al. 2002; Michaud et al. 2002; Amano et al. 2004). The dissimilar GL values may be a consequence of different dietary methods used for data collection. They may, however, also reflect true differences in dietary intake between children and adults, implying that a larger amount of carbohydrate-containing foods are eaten by children. In this context, Liu et al. (2002) and van Dam et al. (2000) both found that the mean carbohydrate intake (energy adjusted) of adults $(220 \mathrm{~g} / \mathrm{d}$ and $238 \mathrm{~g} / \mathrm{d}$, respectively) was lower than among the children in the present study ( $312 \mathrm{~g} / \mathrm{d})$. Similarly, data from the nationwide Danish dietary survey demonstrate an approximately $9 \%$ (100 g/d) higher carbohydrate intake among children (4-14 years) than among adults (15-75 years) from milk products, breads, grains, vegetables, fruits, sugars and sweets in total (Fagt et al. 2002). We thus suggest that the higher GL value of the diet of children compared with adults may be a consequence of a higher carbohydrate intake among children rather than a methodological problem.

The dietary assessment method has some incorporated problems, as has the assignment of GI to food items and the calculation of GL. For example, a single $24 \mathrm{~h}$ recall is not adequate to describe the habitual diet of an individual but is considered sufficient for a description of the habitual energy and macronutrient intakes of larger groups of subjects (Livingstone \& Robson, 2000; Lytle et al. 1993; Biró et al. 2002). It has been argued that an accurate assessment of dietary intake may be specifically difficult among children (Livingstone \& Robson, 2000). However, other studies have found that the $24 \mathrm{~h}$ recall method seems valid for assessing dietary intake among children (Lytle et al. 1993). In the present study, the tenth and ninetieth percentile intakes of energy and macronutrients were of the same magnitude as those reported by children (7-10 years) and adolescents (15-18 years) in the Danish nationwide survey (1995) in spite of the fact that the nationwide survey used a $7 \mathrm{~d}$ estimated diet record (Andersen et al. 1996). Hence, the $24 \mathrm{~h}$ recall method used in the present study seems adequate.

Since information on the GI of Danish food is scarce, the assignment of GI values from foreign foods could introduce bias when calculating daily dietary GI and GL. Furthermore, other factors relating to the diet, for example food preparation or amount of fat, may affect the true values of GI and GL for food items, meals and total diet (World Health Organization, 2003). Such biases would possibly attenuate associations between dietary GI (or GL) and body composition, meaning that the present study's positive findings may be of particular significance. The results of the present study may be of great interest, given the general lack of published literature regarding the habitual dietary GI and GL of children, and on associations between dietary GI (or GL) and body composition.

The present study is cross-sectional, and hence temporal relationships cannot be established. Prospective studies will be needed to examine associations between dietary GI (and GL) and the long-term regulation of body composition.

In conclusion, dietary glycaemic values were positively associated with body fatness among 16-year-old Danish boys, whereas no associations were found among 16-year-old girls, potentially because of under-reporting. Associations were also insignificant for the younger girls and boys. Accordingly, the observed differences may depend on a possible non-uniform effect of diets composed of different foods even though having the same overall dietary GI.

\section{Acknowledgements}

We would like to acknowledge the members of the European Youth Heart Study Group for making their data available. The authors wish to acknowledge the helpful assistance from statistician Peder Frederiksen in analysing data. The establishment of the Research Unit for Dietary Studies was financed by the FREJA (Female Researchers in Joint Action) programme of the Danish Medical Research Council. 


\section{References}

Amano Y, Kawakubo K, Lee JS, Tang AC, Sugiyama M \& Mori K (2004) Correlation between dietary glycemic index and cardiovascular disease risk factors among Japanese women. Eur J Clin Nutr 58, 1472-1478.

Andersen LB (1995) A maximal cycle exercise protocol to predict maximal oxygen uptake. Scand J Med Sci Sports 5, 143-146.

Andersen NL, Fagt S, Groth MV, et al. (1996) Danish dietary habits 1995 (In Danish with a summary in English). Copenhagen: Danish Veterinary and Food Administration.

Augustin LS, Franceschi S, Jenkins DJ, Kendall CW \& La Vecchia C (2002) Glycemic index in chronic disease: a review. Eur J Clin Nutr 56, 1049-1071.

Bandini LG, Must A, Cyr H, Andersen SE, Spadano JL \& Dietz WH (2003) Longitudinal changes in the accuracy of reported energy intake in girls 10-15y of age. Am J Clin Nutr 78, 480-484.

Biró G, Hulshof KF, Ovesen L \& Amorim Cruz JA (2002) Selection of methodology to assess food intake. Eur J Clin Nutr 56, S25-S32.

Bouché C, Rizkalla SW, Luo J, Vidal H, Veronese A, Pacher N, Fouquet C, Lang V \& Slama G (2002) Five-week, low-glycemic index diet decreases total fat mass and improves plasma lipid profile in moderately overweight nondiabetic men. Diabetes Care 25, 822-828.

Brage S, Wedderkopp N, Ekelund U, Franks PW, Wareham NJ, Andersen LB \& Froberg K (2004) Objectively measured physical activity correlates with indices of insulin resistance in Danish children. The European Youth Heart Study, (EYHS). Int J Obes Relat Metab Disord 28, 1503-1508.

Brand-Miller JC, Holt SH \& Petocz P (2003) Reply to R Mendosa. Am J Clin Nutr 77, 994-995.

Cole TJ, Bellizzi MC, Flegal KM \& Dietz WH (2000) Establishing a standard definition for child overweight and obesity worldwide: international survey. BMJ 6, 1240-1243.

Council of Europe (1988) Description of anthropometric measurements. In The Eurofit Test Battery, Danish Edition, pp. 67-70 [B Rasmussen, editor]. Strasbourg: Council of Europe.

Fagt S, Matthiesen J, Trolle E, et al. (2002) Danish dietary habits 20002001. Trends in the dietary consumption, purchasing and habits of the Danish population (In Danish with a summary in English). Copenhagen: Danish Veterinary and Food Administration.

Foster-Powell K, Holt SH \& Brand-Miller JC (2002) International table of glycemic index and glycemic load values: 2002. Am J Clin Nutr 76, 5-56.

Hansen HS, Froberg K, Nielsen JR \& Hyldebrandt N (1989) A new approach to assessing maximal aerobic power in children: the Odense School Child Study. Eur J Appl Physiol Occup Physiol 58, 618-624.

Levi F, Pasche C, Lucchini F, Bosetti C \& La Vecchia C (2002) Glycaemic index, breast and colorectal cancer. Ann Oncol 13, 1688-1689.

Lissner L \& Heitmann BL (1995) Dietary fat and obesity: evidence from epidemiology. Eur J Clin Nutr 49, 79-90.

Liu S, Manson JE, Buring JE, Stampfer MJ, Willett WC \& Ridker PM (2002) Relation between a diet with a high glycemic load and plasma concentrations of high-sensitivity C-reactive protein in middle-aged women. Am J Clin Nutr 75, 492-498.

Liu S, Willett WC, Stampfer MJ, Hu FB, Franz M, Sampson L, Hennekens CH \& Manson JE (2000) A prospective study of dietary glycemic load, carbohydrate intake, and risk of coronary heart disease in US women. Am J Clin Nutr 71, 1455-1461.

Livingstone MB \& Robson PJ (2000) Measurement of dietary intake in children. Proc Nutr Soc 59, 279-293.

Ludwig DS (2000) Dietary glycemic index and obesity. J Nutr 130, 280S-283S

Ludwig DS (2002) The glycemic index: physiological mechanisms relating to obesity, diabetes, and cardiovascular disease. JAMA 287, 2414-2423.

Lytle LA, Nichaman MZ, Obarzanek E, Glousky E, Montgomery D, Nicklas T, Zive M \& Feldman H (1993) Validation of 24-hour recalls assisted by food records in third-grade children. The CATCH Collaborative Group. J Am Diet Assoc 93, 1431-1436.

Michaud DS, Liu S, Giovannucci E, Willett WC, Colditz GA \& Fuchs CS (2002) Dietary sugar, glycemic load, and pancreatic cancer risk in a prospective study. J Natl Cancer Inst 94, 1293-1300.

Moller A (1996) Food composition tables (in Danish). [E Saxholt, editor]. Copenhagen: National Food Agency of Denmark.

Pawlak DB, Ebbeling CB \& Ludwig DS (2002) Should obese patients be counselled to follow a low-glycaemic index diet? Yes. Obes Rev 3, 235-243.

Pawlak DB, Kushner JA \& Ludwig DS (2004) Effects of dietary glycaemic index on adiposity, glucose homoeostasis, and plasma lipids in animals. Lancet 364, 778-785.

Prentice AM \& Jebb SA (2001) Beyond body mass index. Obes Rev 2, 141-147.

Riddoch CJ, Edwards DF, van Mechelen W, et al. (2004a) European Youth Heart Study. Protocols Manual. http://www.ensshe.lu/ committees/children/protocol_European_youth_heart_study.pdf

Riddoch CJ, Edwards DF, van Mechelen W, et al. (2004b) European Youth Heart Study. Background. http://www.ensshe.lu/committees/ children/background.pdf

Salmeron J, Ascherio A, Rimm EB, Colditz GA, Spiegelman D, Jenkins DJ, Stampfer MJ, Wing AL \& Willett WC (1997a) Dietary fiber, glycemic load, and risk of NIDDM in men. Diabetes Care 20 , $545-550$.

Salmeron J, Manson JE, Stampfer MJ, Colditz GA, Wing AC \& Willett WC (1997b) Dietary fiber, glycemic load, and risk of non-insulindependent diabetes mellitus in women. JAMA 277, 472-477.

Sardinha LB, Going SB, Teixeira PJ \& Lohman TG (1999) Receiver operating characteristic analysis of body mass index, triceps skinfold thickness, and arm girth for obesity screening in children and adolescents. Am J Clin Nutr 70, 1090-1095.

Sarría A, Garcia-Llop LA, Moreno LA, Fleta J, Morellon MF \& Buero M (1998) Skinfold thickness measurements are better predictors of body fat percentage than body mass index in male Spanish children and adolescents. Eur J Clin Nutr 52, 573-576.

Scaglioni S, Stival G \& Giovannini M (2004) Dietary glycemic load, overall glycemic index, and serum insulin concentrations in healthy schoolchildren. Am J Clin Nutr 79, 339-340.

Slaughter MH, Lohman TG, Boileau RA, Horswill CA, Stillman RJ, Van Loan MD \& Bember DA (1988) Skinfold equations for estimation of body fatness in children and youth. Hum Biol 60, 709-723.

Tanner JM (1962) Growth at Adolescence. Oxford: Blackwell.

van Dam RM, Visscher AW, Feskens EJ, Verhoef P \& Kromhout D (2000) Dietary glycemic index in relation to metabolic risk factors and incidence of coronary heart disease: the Zutphen Elderly Study. Eur J Clin Nutr 54, 726-731.

Wedderkopp N (2001) Atherosclerotic cardiovascular risk factors in Danish children and adolescents. A community based approach with a special reference to physical fitness and obesity, $\mathrm{PhD}$ Thesis, Institute of Sport Science and Clinical Biomechanics, Faculty of Health Sciences, University of Southern Denmark.

Willett W \& Stampfer MJ (1986) Total energy intake: implications for epidemiologic analyses. Am J Epidemiol 124, 17-27.

Wolever TM \& Jenkins DJ (1985) Application of glycaemic index to mixed meals. Lancet $2,944$.

Wolever TM \& Jenkins DJ (1986) The use of the glycemic index in predicting the blood glucose response to mixed meals. Am J Clin Nutr $\mathbf{4 3}$, 167-172.

World Health Organization (2003) Diet, Nutrition and the Prevention of Chronic Diseases: Report of a Joint WHO/FAO Expert Consultation, Geneva, 28 January - 1 February 2002. Geneva: WHO. 